\title{
Diversidad genética de la población de gatos domésticos (Felis catus) de la ciudad de Mompox, Bolívar, mediante marcadores del pelaje
}

\author{
GENETIC DIVERSITY OF THE POPULATION OF DOMESTIC CATS (Felis catus) OF THE CITY \\ OF MOMPOX, Bolivar, BY COAT MARKERS
}

Enrique Pardo ${ }^{1,2}$, Andrés Castro ${ }^{1}$, Mauricio Begambre ${ }^{1}$

\section{Resumien}

Con el objetivo de analizar la diversidad genética de la población de gatos (Felis catus) de la ciudad colombiana de Mompox, Bolívar, se estudiaron los genes: Orange, Agouti, Tabby, Dilution, Long Hair, Spotting White Dominant White, Manx y Siames para 200 gatos en cinco barrios de la ciudad. Mompox presentó frecuencias alélicas que oscilaron entre 0.678 y 0.003 para los marcadores Non-agouti y Manx, respectivamente. Los marcadores Spotting White y Long Hair mostraron la mayor diversidad; el marcador Orange presentó desequilibrio en dos poblaciones: Seis de Agosto y Faciolince, mientras que Spotting White se mantuvo en equilibrio de Hardy-Weinberg. Se halló una alta diferenciación genética entre las poblaciones $\left(\mathrm{G}_{\mathrm{ST}}=0.1283\right)$. En Mompox se presentó una asociación de las poblaciones atendiendo a la fecha de sus fundaciones. En el Caribe colombiano, las poblaciones se agruparon conforme a los estamentos políticos erigidos desde la colonización y conforme a las épocas de las fundaciones de las ciudades; además, se agruparon por su cercanía geográfica.

Palabras clave: Felis catus; genes; frecuencias alélicas; equilibrio Hardy-Weinberg; distancia genética

\section{ABSTRACT}

The study was conducted to analyze the genetic diversity of the population of cats (Felis catus) of the Colombian city of Mompox, Bolívar. The following genes were studied: Orange, Agouti, Tabby, Dilution, Long Hair, Spotting White Dominant White, Manx and Siames for 200 cats in five neighborhoods of the city. Mompox presented

${ }^{1}$ Departamento de Biología, Facultad de Ciencias Básicas, Universidad de Córdoba, Colombia

${ }^{2}$ Email: epardop@correo.unicordoba.edu.co

Recibido: 11 de octubre de 2017

Aceptado para publicación: 15 de febrero de 2018 
allelic frequencies that ranged between 0.678 and 0.003 for the Non-agouti and Manx markers, respectively. The Spotting White and Long Hair markers showed the greatest diversity; the Orange marker presented disequilibrium in two populations: Seis de Agosto and Faciolince, while Spotting White maintained a Hardy-Weinberg equilibrium. A high genetic differentiation was found between the populations $\left(\mathrm{G}_{\mathrm{ST}}=0.1283\right)$. In Mompox an association of the populations was presented attending the date of their foundations. In the Colombian Caribbean, the populations were grouped according to the political estates erected since the colonization and according to the times of the foundations of the cities; In addition, they were grouped by their geographical proximity.

Key words: Felis catus; genes; allelic frequencies; Hardy-Weinberg equilibrium; genetic distance

\section{INTRODUCCIÓN}

El gato doméstico (Felis catus) desciende de los cruces entre razas silvestres europeas (Felis silvestris silvestris) y africanas ( $F$. s. libyca), y se encuentra entre los más eficientes felinos depredadores de presas medianas y pequeñas (Yamaguchi et al., 2004). Gracias a sus características adaptativas y a su relación con los seres humanos están ampliamente distribuidos en una gran variedad de hábitats (Ruiz et al., 2005).

Los gatos presentan polimorfismos atendiendo al color, el patrón y la textura de la capa, marcadores de fácil identificación; variables generadas por mutaciones que por protección humana (selección positiva) se han conservado en las poblaciones (Fang et al., 2009). Estos marcadores fenotípicos constituyen una valiosa herramienta para analizar la estructura genética de las poblaciones por su bajo costo de muestreo, fácil manipulación e identificación y rápida obtención de resultados. Los estudios de las poblaciones de gatos son importantes para revelar su historia evolutiva y para la construcción de hipótesis filogenéticas sobre las relaciones inter- e intra-poblacionales (Pardo et al., 2016).
La ciudad de Mompox, en el departamento de Bolívar (Colombia) está ubicada en una isla atravesada por caños y por el río Magdalena, arteria fluvial que durante siglos fue el eje que comunicó al país y contribuyó a su desarrollo económico. El tráfico fluvial de la región tenía como epicentro a Mompox y era una de las rutas utilizadas durante la colonización española (Franco, 2009). A finales del siglo XIX se fue reduciendo el trasporte fluvial y comercial, ya que la variabilidad en el curso de las fuentes de agua hizo que las rutas se alteren de acuerdo con las condiciones climáticas. Además, con la llegada del transporte terrestre a Mompox a principios del siglo $\mathrm{XX}$, el comercio fluvial tuvo un serio decaimiento (Barrios, 2013).

Las características geográficas pudieron haber incidido en la dinámica de la población y en la diversidad del gato doméstico en la ciudad de Mompox, debido a que su condición insular podría generar cambios en las frecuencias génicas (Caujapé, 2006). Además de determinar el perfil genético de la población de gatos de la ciudad de Mompox, se busca determinar el posible efecto de la extensión territorial en la diversidad genética, ya que la población está ubicada en una isla que puede ser susceptible a eventos estocásticos como la deriva génica (Peñuela 
y Cárdenas, 2015). Por estas razones el presente estudio tuvo como objetivo analizar la diversidad genética de la población de gatos (Felis catus) de la ciudad de Mompox mediante marcadores del pelaje.

\section{Materiales y Métodos}

La investigación se realizó en la ciudad de Mompox ( $\left.9^{\circ} 142573 \mathrm{Ny} 74^{\circ} 252053 \mathrm{~W}\right)$, departamento de Bolívar, Colombia, la cual presenta una extensión urbana de $21.7 \mathrm{~km}^{2} \mathrm{y}$ una temperatura promedio de $31^{\circ} \mathrm{C}$ (Vélez, 2013).

Se realizaron muestreos tipo incidental mediante observación directa en cinco barrios: La Granja, Faciolince, Seis de Agosto, Centro y Nuevo Horizonte. Se hizo una clasificación fenotípica de los 200 individuos adultos encontrados. La información de campo del estudio en el total de barrios se recolectó en cinco días y en cada barrio el muestreó se realizó en un día para evitar el remuestreo. Se indagó la presencia de gatos en cada casa y bajo el consentimiento de los dueños se tomaron los datos de la presencia o ausencia de cada uno de los marcadores autosómicos non-Agouti(a), Blotched tabby (Tb), Dilution (d), Long hair (l), Spotting white (S), Dominant white (W), Manx (X), Siames $(N)$ y del locus ligado al sexo Orange $(O)$ (Cuadro 1). Asimismo, se hicieron registros fotográficos para cada individuo.

Para el estudio de la diversidad genética de las poblaciones de los gatos se tuvieron en cuenta los siguientes marcadores fenotípicos (Cuadro 1) propuestos por el Committee on Standardized Genetic Nomenclature for Cats (1968): O (Orange. Carácter ligado al sexo) y los loci autosómicos $A$ (A, a; Agouti vs. non-Agouti), $T$ ( Ta, $t+$, tb; Abyssinian tabby vs. Mackerel o atigrado vs. Blotched tabby), D (D, $d$; full color vs. Dilution), $L$ ( $L, l$; pelo corto vs. pelo largo), $S(S+, S$; no manchado de blanco vs. manchado de blanco), $W(w+, W$; color normal vs. Dominant White $), X(x, X$; cola larga vs cola corta o sin cola), $N(n, N$; no oscuro en el hocico vs. oscuro en el hocico).

La estimación de las frecuencias alélicas, la diversidad genética, el flujo génico, el equilibrio Hardy-Weinberg y la distancia genética se realizaron a través del programa PopGene 1.31 (Yeh et al., 1999). Así mismo, se correlacionaron las frecuencias alélicas de los gatos de la ciudad de Mompox con reportes de frecuencias alélicas de ciudades de la región caribe colombiana mediante la distancia genética de Nei (Nei, 1972), con las cuales se graficaron árboles filogenéticos UPGMA con el software MEGA 7 (Kumar et al., 2016).

\section{Resultados}

\section{Frecuencias Alélicas}

El gen Non-agouti fue el de mayor frecuencia en la población (0.678), mientras que los loci Long Hair y Spotting White presentaron frecuencias de 0.539 y 0.523 , respectivamente (Cuadro 2).

Los alelos que expresan los rasgos Dominat White, Manx y Siames en Mompox presentaron las menores frecuencias alélicas, hallándose estos tres marcadores en el barrio de La Granja, mientras que Blochet Tabby no fue observado en las poblaciones.

\section{Diversidad Genética}

La heterocigosidad total en la población de Mompox (Cuadro 3), mostró una media de $\mathrm{H}_{\mathrm{T}}=0.2259$, siendo los marcadores Spoting White $\left(\mathrm{H}_{\mathrm{T}}=0.4999\right)$, Long Hair $\left(\mathrm{H}_{\mathrm{T}}\right.$ $=0.4687)$ y Agouti $\left(\mathrm{H}_{\mathrm{T}}=0.452\right)$ los que presentaron mayor diversidad y $\operatorname{Manx}\left(\mathrm{H}_{\mathrm{T}}=\right.$ 0.0067) quien mostró menor diversidad. 
Cuadro 1. Descripción de los nueve genes estudiados (Ruiz y Álvarez, 2003; Paredes, 2010)

\begin{tabular}{ccl}
\hline Locus & Alelos & Característica \\
\hline$O$ (gen ligado al sexo) & $O$ & Silvestre; pigmentación no naranja \\
& $O$ & $\begin{array}{l}\text { Mutante; presenta pigmentación naranja; epistático } \\
\text { para la detección del locus } A\end{array}$ \\
A (gen autosómico) & $A$ & $\begin{array}{l}\text { Silvestre; color Agouti, el pelaje está dividido en } \\
\text { bandas o franjas de distintos colores }\end{array}$ \\
& $A$ & Mutante; color No-agouti; un mismo color; no se \\
& & observan bandas o franjas; epistático para la \\
& observación del locus T
\end{tabular}

O: Orange; a: Non-agouti; $t^{b}$; Blotched Tabby; d: Dilution; I: Long Hair; s: Spotting White; W: Dominant White; M: Manx; N: Siames

La diversidad genética dentro de las poblaciones reveló un $\mathrm{H}_{\mathrm{S}}=0.221$ y la diversidad entre las poblaciones un $\mathrm{D}_{\mathrm{ST}}=0.029$. El coeficiente de diversidad genética $\left(\mathrm{G}_{\mathrm{ST}}=0.128\right)$ indicó que el $12.83 \%$ de la diversidad genética se encuentra entre las poblaciones y el $87.17 \%$ dentro de las poblaciones. El valor $\mathrm{Nm}$ (3.3), se encontró entre 1 y 4 , lo cual indica que existe un flujo génico moderado entre las poblaciones locales estudiadas (Piñero et al., 2008).

\section{Equilibrio de Hardy-Weinberg}

A nivel global, se encontró ausencia de equilibrio de Hardy-Weinberg para el marcador Orange (0.000), así como para las poblaciones Seis de Agosto (0.049) y Faciolince (0.002), mientras que el marcador Spoting White se mantuvo en equilibrio de HardyWeinberg (Cuadro 4). 
Cuadro 2. Frecuencia alélica de los marcadores en las poblaciones de gatos domésticos en la ciudad de Mompox, Bolívar

\begin{tabular}{lcccccccccc}
\hline Poblaciones & $\mathrm{N}^{\circ}$ & $O$ & $a$ & $t^{b}$ & $D$ & $l$ & $S$ & $W$ & $M$ & $N$ \\
\hline Seis de Agosto & 30 & 0.300 & 0.447 & 0.000 & 0.105 & 0.183 & 0.333 & 0.000 & 0.000 & 0.051 \\
La Granja & 30 & 0.103 & 0.809 & 0.000 & 0.017 & 0.000 & 0.500 & 0.017 & 0.017 & 0.017 \\
Centro & 37 & 0.347 & 0.553 & 0.000 & 0.102 & 0.329 & 0.542 & 0.014 & 0.000 & 0.000 \\
Nuevo Horizonte & 51 & 0.255 & 0.721 & 0.000 & 0.116 & 0.464 & 0.510 & 0.010 & 0.000 & 0.020 \\
Faciolince & 52 & 0.350 & 0.741 & 0.000 & 0.081 & 0.899 & 0.647 & 0.010 & 0.000 & 0.000 \\
\hline Promedio & & 0.281 & 0.678 & 0.000 & 0.088 & 0.539 & 0.523 & 0.010 & 0.003 & 0.015 \\
\hline
\end{tabular}

O: Orange; a: Non-agouti; $t^{b}$ : Blotched Tabby; $d$ : Dilution; I: Long Hair; s: Spotting White; W: Dominant White; M: Manx; N: Siames

Cuadro 3. Distribución de la diversidad genética en los marcadores genéticos en gatos domésticos en la ciudad de Mompox, Bolívar

\begin{tabular}{lccccc}
\hline & $\mathrm{H}_{\mathrm{T}}$ & $\mathrm{H}_{\mathrm{S}}$ & $\mathrm{D}_{\mathrm{ST}}$ & $\mathrm{G}_{\mathrm{ST}}$ & $\mathrm{Nm}$ \\
\hline Orange & 0.395 & 0.378 & 0.016 & 0.041 & 11.4 \\
Agouti & 0.452 & 0.416 & 0.035 & 0.078 & 5.8 \\
Tabby & 0.000 & 0.000 & 0.000 & 0.000 & 0.0 \\
Dilution & 0.155 & 0.152 & 0.002 & 0.016 & 30.1 \\
Long Hair & 0.468 & 0.283 & 0.184 & 0.394 & 0.7 \\
Spoting White & 0.499 & 0.479 & 0.020 & 0.040 & 11.7 \\
White & 0.019 & 0.019 & 0.000 & 0.003 & 154.4 \\
Manx & 0.006 & 0.006 & 0.000 & 0.013 & 36.5 \\
Siames & 0.034 & 0.034 & 0.000 & 0.020 & 24.2 \\
\hline Media & 0.254 & 0.221 & 0.029 & 0.128 & 3.3 \\
\hline
\end{tabular}

$\mathrm{H}_{\mathrm{T}}$ : diversidad genética total; $\mathrm{H}_{\mathrm{s}}$ : diversidad dentro de las poblaciones; $\mathrm{DsT}$ : diversidad entre poblaciones; Gst: coeficiente de diversidad genética; Nm: Flujo génico

Cuadro 4. Análisis de equilibrio de Hardy-Weinberg en los locus Orange y Spotting White en gatos domésticos en la ciudad de Mompox, Bolívar

\begin{tabular}{lcccccc}
\hline & Mompox & $\begin{array}{c}\text { Seis de } \\
\text { Agosto }\end{array}$ & La granja & Centro & $\begin{array}{c}\text { Nuevo } \\
\text { Horizonte }\end{array}$ & Faciolince \\
\hline Orange & 0.0000 & 0.0495 & 0.9354 & 0.0674 & 0.2468 & 0.0019 \\
Spotting White & 0.2982 & 0.8607 & 0.1309 & 0.9205 & 0.2422 & 0.3569 \\
\hline
\end{tabular}


Cuadro 5. Matriz de distancias genética $\left(D_{N e i}\right)$ entre las poblaciones de gatos domésticos en la ciudad de Mompox, Bolívar

\begin{tabular}{|c|c|c|c|c|c|}
\hline Población & Seis de Agosto & La Granja & Centro & Nuevo Horizonte & Faciolince \\
\hline Seis de Agosto & $\begin{array}{ll}---- \\
--1\end{array}$ & & & & \\
\hline La Granja & 0.031 & ----- & & & \\
\hline Centro & 0.011 & 0.031 & ------ & & \\
\hline Nuevo Horizonte & 0.027 & 0.033 & 0.008 & ------ & \\
\hline Faciolince & 0.102 & 0.125 & 0.053 & 0.030 & -------- \\
\hline
\end{tabular}
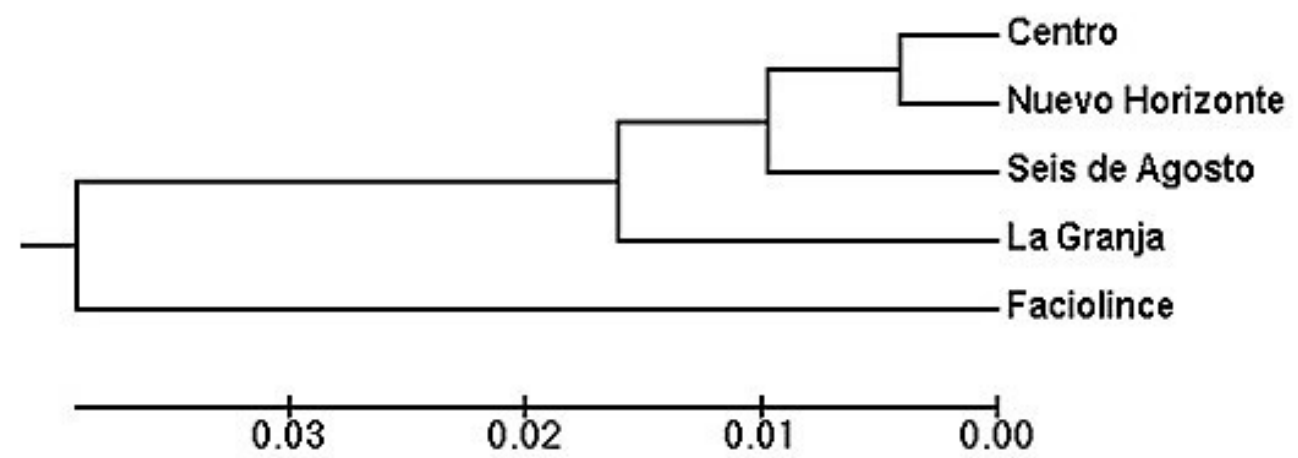

Figura 1. Árbol UPGMA obtenido a partir de las distancias genéticas de Nei (1972) que muestra las relaciones entre las poblaciones de gatos domésticos de la ciudad de Mompox, Bolívar
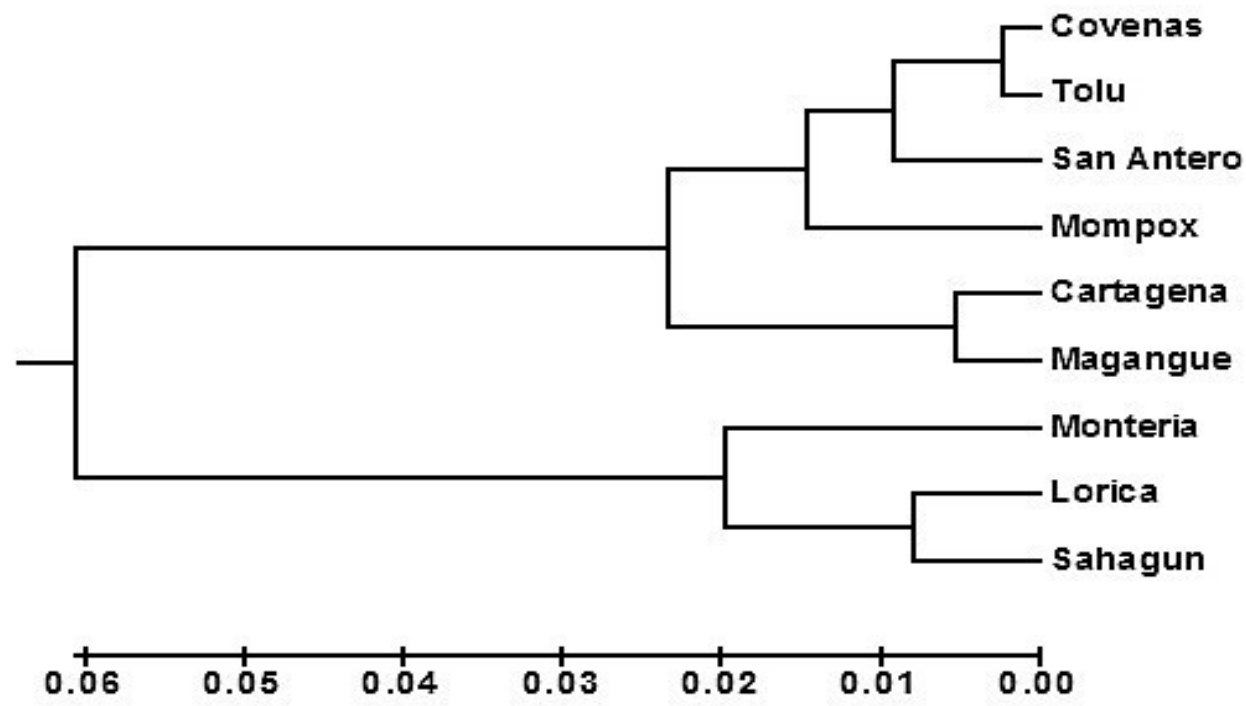

Figura 2. Árbol UPGMA obtenido a partir de la distancia genética de Nei (1972) en las poblaciones de gatos domésticos del Caribe colombiano 


\section{Distancia Genética}

La distancia genética entre las poblaciones ubicó a Nuevo Horizonte y Centro como las más cercanas, mientras que La Granja y Faciolince se comportaron como las más alejadas (Cuadro 5). Asimismo, el dendograma (Figura 1) evidenció la similitud genética entre las poblaciones de Centro y Nuevo Horizonte, a las cuales se asocia las poblaciones de Seis de Agosto y La Granja. Por otro lado, la población Faciolince fue la más alejada.

El dendograma elaborado para el Caribe colombiano (Figura 2) reveló la formación de dos grupos, estando el primero formado por Coveñas, Tolú, San Antero, Mompox, Cartagena y Magangué, y el segundo grupo por Montería, a las cuales se unen Lorica y Sahagún.

\section{Discusión}

Mompox presenta una alta frecuencia del alelo Non-agouti, lo cual podría estar fundamentado en el hecho de que las alternativas alélicas que posibilitan coloraciones más melánicas se verían favorecidas en el medio urbano (Ruiz Álvarez, 2003). Otro factor seria por condiciones ambientales como las altas temperaturas que podrían favorecer tanto la presencia como el aumento de individuos portadores de dicho gen (Causil et al., 2017). Estos resultados fueron mayores a los observados en Lorica, Santa Marta y Montería (Pardo et al., 2014, 2015; Causil et al., 2017) y bajos en relación con Tolú, Cartagena y Magangué (Martínez y Zambrano, 2014; Montes et al., 2015; Pardo et al., 2016).

La frecuencia del alelo Long Hair (Cuadro 2) pudo deberse a la preferencia humana, lo cual altera el pool genético inicial; similar al estudio de Wilkinson et al. (2013) en cerdos europeos, donde la selección ha modificado la coloración del pelaje, composición muscular y tamaño corporal, así como la reproducción y el comportamiento.
La presencia del gen Manx en Mompox puede asociarse con la época de mayor dispersión de este marcador entre los siglos XVIII y XIX (Robbins, 2012) y su baja frecuencia, relacionándose con el aislamiento en que quedó dicha población, luego del abandono del río Magdalena como vía fluvial y comercial (Franco, 2009), lo cual afectó el flujo genético de los gatos de Mompox. Este mismo evento de aislamiento también afectó al alelo Siames.

La ausencia del locus Blochet Tabby en este estudio fue similar a otros reportes (Ruiz y Álvarez, 2008; Pardo et al., 2016) donde se afirma que las frecuencias de este alelo en Latinoamérica resultaron ser bajas o muy bajas.

La diversidad genética total $\left(\mathrm{H}_{\mathrm{T}}\right)$ encontrada en Mompox fue moderada. La mayor parte se encontró dentro de las poblaciones $(\mathrm{Hs})$ y poca entre las poblaciones $\left(\mathrm{D}_{\mathrm{ST}}\right)$, lo cual demuestra que las subpoblaciones en Mompox comparten una gran proporción de la diversidad total. La heterocigosidad total presente fue menor (Cuadro 3) respecto a lo reportado para Cartagena, Santa Marta, y Riohacha (Montes et al., 2015; Causil et al., 2017; Pardo et al., 2017) y mayor a lo reportado en Montería y Lorica (Pardo et al., 2014, 2015).

El elevado valor de $\mathrm{G}_{\mathrm{ST}}$ obtenido (0.128) es de los mayores registrados en Colombia (Martínez y Zambrano, 2014; Montes, et al., 2015; Peña et al., 2015; Causil et al., 2017), lo cual debe estar relacionado con el rápido crecimiento demográfico de Mompox y con la formación de nuevos barrios, lo cual ha provocado una elevada diferenciación entre las poblaciones estudiadas (Caujapé, 2006).

El nivel de flujo genético obtenido permite inferir que las poblaciones se encuentran muy relacionadas, ya que cuando $\mathrm{Nm}$ es mayor a 1 se espera que las poblaciones mantengan conectividad genética, debido a que el flujo genético supera los efectos de la 
deriva e imposibilita la diferenciación local (Montes et al., 2015).

La desviación del equilibrio de HardyWeinberg para el marcador Orange puede estar ocasionada por la existencia de marcadores ligados a algún otro gen afectado por heterosis, efecto Wahlund, mutación, deriva génica o selección humana por cierto tipo de pelaje (Pacheco et al., 2014).

El dendrograma UPGMA, obtenido a partir de los valores de distancia genética de Nei (1972) para las poblaciones estudiadas, relaciona las poblaciones con la fecha de su fundación y con su ubicación geográfica. Además, el valor obtenido para la distancia genética permite concluir que las poblaciones están muy relacionadas.

La asociación encontrada entre Coveñas, Tolú, San Antero, Mompox, Cartagena y Magangué puede relacionarse con la pertenencia de estas al mismo estamento político $\mathrm{y}$ a las fechas de fundación semejantes, al estar dichas poblaciones bajo el mandato de La Provincia de Cartagena entre los años 1533-1810 y luego al Estado Soberano de Bolívar hasta 1886 (Lambis, 2011). La agrupación de las poblaciones Montería, Lorica y Sahagún en el dendograma (Figura 2) puede responder a su cercanía geográfica, a su historia reciente y al elevado flujo génico humano que se da entre estas poblaciones (Montes y Martínez, 2008).

\section{Conclusiones}

- Las frecuencias alélicas de las mutaciones que modifican el patrón y la coloración del pelaje en gatos en la población de Mompox fueron: Orange $=0.281$, Non-agouti $=0.678$, Blochet Tabby $=$ 0.000 , Dilution $=0.088$, Long Hair $=$ 0.539 , Spottin White $=0.523$, Dominant White $=0.010$, Manx $=0.003$ y Siames $=0.015$.
- La población de Mompox presentó mayor diversidad genética $\left(\mathrm{G}_{\mathrm{ST}}=0.1283\right)$ dentro de las poblaciones que entre las poblaciones. También se encontró la existencia de un flujo génico moderado entre las poblaciones. El marcador Orange reveló ausencia de equilibrio de Hardy-Weinberg.

- En Mompox, las poblaciones se asociaron teniendo en cuenta su fecha de fundación y su ubicación geográfica. Asimismo, según la distancia genética se puede indicar que las poblaciones están muy relacionadas.

\section{Literatura Citada}

1. Barrios S. 2013. Un rio que cambia el lugar de las ciudades, el rio Magdalena de Mompox a Magangué. Credencial Historia. [Internet]. Disponible en: http:/ /www.banrepcultural.org/blaavirtual/revistas/credencial/diciembre-2013/un-rioque-cambia

2. Caujapé J. 2006. Brújula para botánicos desorientados en la genética de poblaciones. Las Palmas de Gran Canaria, España: Exegen Ed.133 p.

3. Causil L, Pardo E, Muñoz B. 2017. Estructura genética-poblacional de gatos domésticos (Felis catus), usando marcadores fenotípicos en Santa Marta, Colombia. Rev Logos Cienc Tecnol 8: 182-190. doi: 10.223335/rlct.v8i2.293

4. Commitee on Standardized Genetic Nomenclature for Cats. 1968. Standardized genetic nomenclature for the domestic cat. J Hered 59: 39-40.

5. Fang M, Larson G, Ribeiro $\mathrm{H}, \mathrm{Li} \mathrm{N}$, Andersson L. 2009. Contrasting mode of evolution at a coat color locus in wild and domestic pigs. Plos Genetics 5(1). doi: 10.1371/jornal.pgen.1000341

6. Franco L. 2009. Mompox y el río grande de la Magdalena. Rev Gestión Ambiente 12: 119-132. 
7. Kumar S, Stecher G, Tamura K. 2016. MEGA7: Molecular evolutionary genetics analysis version 7.0 for bigger datasets. Mol Bio Evol 33: 1870-1874. doi: 10.1093/molbev/msw054

8. Lambis D. 2011. División territorial en Bolívar: la lucha por la autonomía y la construcción de identidades territoriales. Tesis de pregrado. Colombia: Univ. de Cartagena. 227 p.

9. Martínez M, Zambrano J. 2014. Descripción de la frecuencia de algunos alelos mutantes mediante genes asociados a la coloración del pelaje de gato doméstico (Felis catus) en el caribe: Tolú, Coveñas-Sucre, San Antero-Córdoba. Tesis de pregrado. Colombia: Univ. de Córdoba. $82 \mathrm{p}$.

10. Montes R, Martínez A. 2008. El Sinú y las sabanas. Cuadernos de Literatura del Caribe e Hispanoamérica 8: 11-24.

11. Montes Y, Barrios Y, Pardo E. 2015. Análisis de la variabilidad genética de las poblaciones de gatos domésticos (Felis catus) mediante genes del pelaje en Cartagena, Colombia. Rev Acad Col Cie Exa Fís Nat 39: 503-513. doi: 10.18257/ raccefyn. 240

12. Nei M. 1972. Genetic distance between populations. Amer Nat 106: 283-292. doi: 10.1086/282771

13. Pacheco E, Arias D, Ojeda Z, Romero $H$. 2014. Diversidad y estructura genética de accesiones de palma de aceite (Elaeis guineesis Jacq.) provenientes de Camerún. Rev Colomb Biotecnol 16: 57-67.

14. Pardo E, Morales J, Cavadía T. 2014. Estudio de la diversidad genética de la población de gato doméstico (Felis catus) en Montería, Colombia. Bistua 12(2): 35-47.

15. Pardo E, Causil L, Rodríguez A. 2015. Estudio de la diversidad genética de gato doméstico (Felis catus) mediante genes asociados al color del pelaje en LoricaCórdoba, Colombia. Arch Zootec 64: 389-395.
16. Pardo E, Montes Y, Cardales Y. 2016. Variabilidad genética del gato doméstico (Felis catus) en Magangué, Bolívar, Colombia. Rev Inv Vet Per 27: 277-287. doi: 10.15381/rivep.v27i2.11661

17. Pardo E, Causil L, Muñoz B. 2017. Perfil genético de la población de gatos (Felis catus) en Riohacha, La Guajira, mediante genes de pelaje. Rev Fac Cien Bás 13: 128-132. doi: 10.18359/rfcb.2750

18. Paredes E. 2010. Descripción de la frecuencia de algunos alelos mutantes de la capa de los gatos domésticos (Felis catus) de la ciudad de Iquique. Tesis de pregrado. Chillán, Chile: Univ. de Concepción. 52 p.

19. Peña-Cruz AF, Arango $S$, Patiño AM, Bedoya M, Rodríguez AO, et al. 2015. Genetic analysis of the cat population of north and south of Cali, Colombia. Act Biol Colomb 20: 109-116. doi: 10.15446/ abc.v20n1.41610

20. Peñuela M, Cárdenas H. 2015. Marcadores genéticos del pelaje en gatos domésticos de Capurganá-Colombia. Mom Cienc 9(1): 28-33.

21. Piñero D, Caballero M, Cabrera T, Canteros H, Casas A, Castañeda A, Castillo $A$, et al. 2008. La diversidad genética como instrumento para la conservación y el aprovechamiento de la biodiversidad: estudios en especies mexicanas. En: Capital Natural de México. Conocimiento actual de la biodiver-sidad. Vol 1. México: CONABIO. p. 437-494.

22. Robbins N. 2012. Domestic cats: their history, breeds and other facts. USA: Createspace. 290 p.

23. Ruiz G, Alvarez D. 2003. Posible origen europeo de seis poblaciones latinoamericanas de gatos y no existencia de paralelismo con el modelo colonizador británico al utilizar genes del pelaje y microsatélites. Act Zool Mex 89: 261-286.

24. Ruiz G, Alvarez D, Shostell J. 2005. Population genetic analysis of cat populations from Mexico, Colombia, Bolivia, and the Dominican Republic: 
identification of different gene pools in Latin America. J Genet 84: 147-171.

25. Ruiz G, Alvarez D. 2008. A biogeographical population genetics perspective of the colonization of cats in Latin America and temporal genetic changes in Brazilian cat populations. Gen Mol Biol 31: 772-782. doi: 10.1590/S141547572008000400026

26. Vélez J. 2013. El conocimiento local en la gestión del riesgo de inundaciones: comunidades del municipio de Mompós Bolívar. Tesis Doctoral. Medellín, Colombia: Univ. Nacional de Colombia. $140 \mathrm{p}$.

27. Wilkinson S, Lu ZH, Megens HJ, Archibald AL, Haley C, Jackson J,
Groenen M, et al. 2013. Signatures of diversifying selection in European pig breeds. Plos Genet 9. doi: 10.1371/ journal.pgen.1003453

28. Yamaguchi A, Ono T, Nasu S, Miyake K, Mibu K, Shinjo T. 2004. Real-space observation of current-driven domain wall motion in submicron magnetic wires. Phys Rev Let 92: 077205. doi: 10.1103/PhysRevLett.92.077205

29. Yeh FC, Rong-cai Yang, Boyle T. 1999. POPGENE Version 1.31. Microsoft Windows-based freeware for population genetic analysis. Canada: University of Alberta. $28 \mathrm{p}$. 